\title{
Robust Fault Estimation Using Relative Information in Linear Multi-Agent Networks
}

\author{
Prathyush P. Menon and Christopher Edwards
}

\begin{abstract}
In this paper a robust fault estimation method, based on sliding mode observers, is proposed for a collection of agents undertaking a shared task and exchanging only relative information over a communication network. Since the 'system of systems' formed by the agents is not observable with respect to relative sensing information, by appropriate transformations and scalings of the inputs and outputs of the actual system, a meaningful observable subsystem is created. For this new subsystem, after modal decomposition based on the associated Laplacian, decoupled sliding mode observers, depending only on the individual node level dynamics of the network, can be created exploiting an existing design philosophy. These collectively form a centralized fault estimation scheme for the original system.
\end{abstract}

Index Terms-Multi agent networks, Faults, Sliding mode observer

\section{INTRODUCTION}

The motivation for the present work is to facilitate an increased level of autonomy in the framework of a 'systems of systems' i.e. a group of dynamic systems cooperating over a network, as reported in [1], [2], to perform a shared common objective. In such an architecture a single undetected fault in one agent could have a severe undesirable impact on the overall system performance. In this paper the network represents an interconnection topology over which individual systems exchange information. Systems operated in such cooperative and distributed environments, making use of relative information exchange, are prevalent in many areas of research, for example mobile robots, cooperating UAV team operations (surveillance and reconnaissance), formation flying of UAV's and satellites, vehicle platoons, and distributed state estimation applications such as localisation: for details, see [1], [3], [4]. These systems, with increasing size and complexity, are intended to operate in a highly autonomous way. Hence, to ensure safe and reliable operations, these systems, requiring a high level of autonomy, must be provided with a state monitoring and fault detection capability.

Since the graph describing the topology of the information network is the lynch-pin, according to [5], broadly speaking, state agreement, synchronisation and consensus problems can be considered from an identical point of view. In [6], an adaptive sliding mode observer is designed for synchronisation of coupled nonlinear systems while adaptive sliding mode

Centre for Systems, Dynamics and Control, College of Engineering, Mathematics and Physical Sciences, University of Exeter, Exeter, EX4 4QF, UK. (email: P.M.Prathyush@exeter.ac.uk, C.Edwards@exeter.ac.uk) control has been applied in [7] for synchronisation. Both of these applications adopt a master-slave framework. Recently, a second order sliding mode observer together with a bank of wavelet networks has been designed specifically for online monitoring and fault detection in satellite formations in the presence of uncertainty [8]. In [9], an observer-controller pair is proposed for a single unicycle mobile robot. Reference [10] considers a similar problem giving a sufficient condition for observability, and proposes an Extended Kalman Filter (EKF) scheme for the localization problem. The EKF estimates the states of the leader-follower formation from the measurements and control signals computed at the leader level. References [8]-[11] give further examples of the use of observer schemes in complex applications. Estimation over graphs from relative measurements are discussed in [12], and recently in [13], distributed unknown input observers have been designed for fault estimation in power networks.

Limited research has taken place in the field of fault detection and isolation (FDI) for large scale systems [14]-[16]. Although some results in this field were published in the 1980's, the literature is sparse. Using overlapping decomposition, the work described in [17] proposed a fault detection approach for discrete linear large scale systems. Subsequently the detection of abrupt faults in discrete systems was considered in [18] and decentralised fault detection schemes have also been proposed [15], [16]. More recently Patton and co-workers have proposed a framework for fault detection in networked systems [19], [20]. A similar problem to that investigated in [21] is considered in this paper - the methodology however is entirely different. The work in [21] examines how the fault detection and isolation ideas of [22] can be extended to a group of agents exchanging relative information. In this paper fault estimation (rather than FDI) will be pursued. Fault estimation is a more stringent requirement compared to traditional FDI problems which only seek to raise an alarm when a fault is present in the system (and then isolate the source). Estimating faults is more useful since the magnitude and 'shape' of the fault is reproduced. As a consequence it is easier to distinguish the presence of intermittent faults from persistent ones and also directly assess their severity. Fault estimation methods typically require stronger assumptions than residual based FDI methods, but have advantages in terms of performance.

Many monitoring and fault estimation schemes are based on model based techniques [23], and in general, the focus is on centralised monitoring schemes which require all the measurements in the entire large scale system. The contribution of this paper is to propose a framework which, although it is centralised in its implementation, the design of the observer 
components are entirely decentralised. From a synthesis point of view this has the benefit that the number of decision variables in the associated optimisation problem is of the order of the dimension of a single node, and not of the system of systems. Furthermore, the proposed method requires only access to relative measurements and the input signal. This paper builds on earlier work on sliding mode observers for fault estimation originally proposed in [24].

The notation used in this paper is standard. The set of real numbers is denoted by $\mathbb{R}$. The set of real-valued vectors of length $m$ is given by $\mathbb{R}^{m}$. The set of arbitrary real-valued $m \times$ $n$ matrices is given by $\mathbb{R}^{m \times n}$. The expression $\operatorname{Col}($.) denotes a column vector and $\operatorname{Diag}($.$) denotes a diagonal matrix. The$ symbols $\mathcal{N}(\cdot)$ and $\mathcal{R}(\cdot)$ represent the null space and range space of a matrix respectively and $\mathbf{1}$ represents a column vector of unity elements.

\section{SYSTEM DESCRIPTION}

This paper, considers a group of dynamical systems interacting to achieve a collective objective. The overall system comprises interconnections of $N$ identical dynamical systems indexed as $1,2, \ldots, N$. Each system has access to relative external measurements associated with certain other dynamical systems which it can sense or interact with. In this paper whenever there exists interaction, bidirectional communication is assumed and the configuration is viewed from an undirected graph perspective [1]. The graph will have $N$ vertices/nodes and each represents an $n$-dimensional dynamical system. An edge in the graph indicates an interaction between the two corresponding dynamical systems. The dynamics of the $i^{t h}$ individual node are given by

$$
\begin{aligned}
\dot{x}_{i}(t) & =A x_{i}(t)+B u_{i}(t)+D f_{i}(t)+E \xi_{i}(t) \\
z_{i j}(t) & =C\left(x_{i}(t)-x_{j}(t)\right), \quad j \in \mathcal{J}_{i}
\end{aligned}
$$

where $x_{i} \in \mathbb{R}^{n}$ and $u_{i} \in \mathbb{R}^{m}$ represent the states and the control inputs of the $i^{t h}$ node. The unknown signals $\xi_{i}(t)$ represent uncertainty or exogenous disturbances which are bounded with a finite $\mathcal{L}_{2}$ norm. The matrices are $A \in \mathbb{R}^{n \times n}$, $B \in \mathbb{R}^{n \times m}, C \in \mathbb{R}^{p \times n}, D \in \mathbb{R}^{n \times q}$ and $E \in \mathbb{R}^{n \times r}$. In (1) the signal $f_{i}(t)$ represents a fault: if $f_{i}(t) \equiv 0$, the system is fault free, and if $f_{i}(t) \neq 0$ then a fault exists in the $i^{t h}$ node. The quantities $z_{i j} \in \mathbb{R}^{p}$ represent relative measurements associated with the other dynamical systems which the $i^{\text {th }}$ node can sense. The set $\mathcal{J}_{i} \subset\{1, \ldots, N\} /\{i\}$ indicates the dynamical systems with which the $i^{\text {th }}$ dynamical system interacts. Here, it is assumed that none of the $\mathcal{J}_{i}$ are empty so that every dynamical system interacts with at least one other. Define an aggregated relative information measurement associated with the $i^{\text {th }}$ node by

$$
z_{i}=\sum_{j \in \mathcal{J}_{i}} C\left(x_{i}-x_{j}\right)
$$

This notation is consistent with that used in [1] and [2]. The Laplacian of the graph $\mathcal{G}$ formed from the interacting agents, which will be written as $L \in \mathbb{R}^{N \times N}$, is defined as follows:

$$
\begin{aligned}
L_{i i} & =\left|\mathcal{J}_{i}\right| \\
L_{i j} & = \begin{cases}-1, & j \in \mathcal{J}_{i} \\
0 & j \notin \mathcal{J}_{i}\end{cases}
\end{aligned}
$$

where $\left|\mathcal{J}_{i}\right|$ denotes the cardinality of $\mathcal{J}_{i}$ and represents the degree of the node. The symmetric matrix $L$ is always rank deficient and positive semi-definite in the case of undirected graphs. The smallest eigenvalue of $L$ is zero and the corresponding eigenvector is given by $\mathbf{1}$ [25].

Assumption 1: The triple $(A, D, C)$ is assumed to be a minimal realisation of the $i^{t h}$ node dynamics, with $D$ and $C$ of full column and row rank respectively where $p \geq q$.

Assumption 2: The system $(A, D, C)$ is minimum phase.

Assumption 3: $\operatorname{rank}(C D)=q$ for every dynamical system

Assumption 4: The function $f_{i}(t)$ represents an unknown time varying fault in the $i^{\text {th }}$ system which is bounded such that $\left\|f_{i}(t)\right\| \leq \beta_{0}$, where $\beta_{0}$ is known a-priori. ${ }^{1}$

The overall system (4)-(5) at the network level can be conveniently represented as:

$$
\begin{aligned}
\dot{x}(t)= & \underbrace{\left(I_{N} \otimes A\right)}_{A_{N}} x(t)+\underbrace{\left(I_{N} \otimes B\right)}_{B_{N}} u(t)+\underbrace{\left(I_{N} \otimes D\right)}_{D_{N}} f(t) \\
& +\underbrace{\left(I_{N} \otimes E\right)}_{E_{N}} \xi(t) \\
z= & \underbrace{(L \otimes C)}_{C_{N}} x(t)
\end{aligned}
$$

where $x:=\operatorname{Col}\left(x_{1}, \ldots, x_{N}\right), \quad u:=\operatorname{Col}\left(u_{1}, \ldots, u_{N}\right)$, $f:=\operatorname{Col}\left(f_{1}, \ldots, f_{N}\right), \quad \xi:=\operatorname{Col}\left(\xi_{1}, \ldots, \xi_{N}\right)$ and $z:=$ $\operatorname{Col}\left(z_{1}, z_{2}, \ldots, z_{N}\right)$. The objective is to design an observer scheme to reconstruct the faults in the system from the relative measurements $z$.

Lemma 1: The pair $\left(L \otimes C, I_{N} \otimes A\right)$ associated with the dynamical system at a network level in (6) - (7), defined with respect to the relative measurement signals, is not observable.

Proof: The pair $\left(L \otimes C, I_{N} \otimes A\right)$ is observable if and only if the associated Popov-Belevitch-Hautus (PBH) matrix pencil for the system in (6)-(7), given by

$$
\mathcal{O}(s):=\left[\begin{array}{c}
(L \otimes C) \\
s I_{N n}-\left(I_{N} \otimes A\right)
\end{array}\right]=\left[\begin{array}{c}
(L \otimes C) \\
I_{N} \otimes\left(s I_{n}-A\right)
\end{array}\right]
$$

is full column rank for all $s \in \mathbb{C}$ [26]. Since $L$ is symmetric positive semi-definite, by spectral decomposition $L=V \Lambda V^{\mathrm{T}}$, where the orthogonal matrix $V \in \mathbb{R}^{N \times N}$ constitutes the eigenvectors of $L$, and $\Lambda=\operatorname{Diag}\left(\lambda_{1}, \ldots \lambda_{N}\right) \in \mathbb{R}^{N \times N}$ where the eigenvalues of $L$ are ordered such that $\lambda_{1}=0<\lambda_{2} \leq$ $\ldots \leq \lambda_{N}$. Since $L$ is symmetric it is guaranteed to have $N$ real eigenvalues. Define two orthogonal scaling matrices

$$
\mathbf{T}_{l}:=\left[\begin{array}{cc}
\left(V^{\mathrm{T}} \otimes I_{p}\right) & \mathbf{0} \\
\mathbf{0} & \left(V^{\mathrm{T}} \otimes I_{n}\right)
\end{array}\right] \quad \text { and } \quad \mathbf{T}_{r}:=\left(V \otimes I_{n}\right)
$$

Pre and post multiply the PBH matrix $\mathcal{O}(s)$ in (8) using $\mathbf{T}_{l}$ and $\mathbf{T}_{r}$ to create $\mathbf{T}_{l} \mathcal{O}(s) \mathbf{T}_{r}$. Since the scaling matrices $\mathbf{T}_{l}$ and $\mathbf{T}_{r}$ are orthogonal, $\mathbf{T}_{l} \mathcal{O}(s) \mathbf{T}_{r}$ is full rank if and only if $\mathcal{O}(s)$ is full rank for all $s \in \mathbb{C}$. Since $V^{\mathrm{T}} V=I_{N}$ and $V^{\mathrm{T}} L V=\Lambda$, it follows from the properties of Kronecker products that $\left(V^{\mathrm{T}} \otimes\right.$ $\left.I_{p}\right)(L \otimes C)\left(V \otimes I_{n}\right)=\left(V^{\mathrm{T}} L V \otimes C\right)=(\Lambda \otimes C)$ and similarly

\footnotetext{
${ }^{1}$ It is reasonable to assume that at least for a finite time interval after its initial occurrence the fault $f_{i}(t)$ remains bounded, this allows a reasonable time window in which detection and diagnosis can take place.
} 
$\left(V^{\mathrm{T}} \otimes I_{n}\right)\left(I_{N} \otimes\left(s I_{n}-A\right)\right)\left(V \otimes I_{n}\right)=\left(I_{N} \otimes\left(s I_{n}-A\right)\right)$. From these equalities:

$$
\mathbf{T}_{l} \mathcal{O}(s) \mathbf{T}_{r}=\left[\begin{array}{c}
(\Lambda \otimes C) \\
I_{N} \otimes\left(s I_{n}-A\right)
\end{array}\right]
$$

Since $\Lambda$ is diagonal, $(\Lambda \otimes C)$ can be written as $\operatorname{Diag}\left(\lambda_{1} C, \ldots, \lambda_{N} C\right)$ and therefore from (9)

$$
\operatorname{rank}\left(\mathbf{T}_{l} \mathcal{O}(s) \mathbf{T}_{r}\right)=\sum_{i=1}^{N} \operatorname{rank}\left(\left[\begin{array}{c}
\lambda_{i} C \\
s I_{n}-A
\end{array}\right]\right)
$$

From Assumption 1 the pair $(C, A)$ is observable, implying $\left[C^{\mathrm{T}}(s I-A)^{\mathrm{T}}\right]^{\mathrm{T}}$ is full column rank. However, $\lambda_{1}=0$ whereas all other $\lambda_{i}, i=2, \ldots N$ are strictly positive. Consequently

$$
\operatorname{rank}\left[\begin{array}{c}
\lambda_{1} C \\
(s I-A)
\end{array}\right]=\operatorname{rank}(s I-A)
$$

and $\operatorname{rank}\left(\mathbf{T}_{l} \mathcal{O}(s) \mathbf{T}_{r}\right)=(N-1) \times n+\operatorname{rank}(s I-A)$. Because $\mathbf{T}_{l} \mathcal{O}(s) \mathbf{T}_{r}$ (and hence $\mathcal{O}(s)$ ) looses rank if $s$ is an eigenvalue of $A$, the pair $\left(L \otimes C, I_{N} \otimes A\right)$ is not observable.

Remark 1: The unobservable modes of $\left(L \otimes C, I_{N} \otimes A\right)$ are the eigenvalues of $A$ and consequently if the system matrix $A$ is stable $\left(L \otimes C, I_{N} \otimes A\right)$ is detectable. The difficulty in estimating the complete state $x$ from the relative measurements $z$ is also reported in [21].

To extract the observable-subspace define a coordinate transformation $x \rightarrow T x=x_{o}$, where $T \in \mathbb{R}^{N n \times N n}$, such that $x_{o}=(\underbrace{T_{s}^{-1} \otimes I_{n}}_{T}) x$ and

$$
T_{s}^{-1}:=\left[\begin{array}{cc}
1 & \mathbf{0} \\
-\mathbf{1}_{N-1} & I_{N-1}
\end{array}\right]
$$

Applying the transformation $T$ to the states $x$ yields

$$
x_{o}=\operatorname{Col}\left(x_{1}, \bar{x}_{2}, \bar{x}_{3}, \ldots, \bar{x}_{N}\right)=\operatorname{Col}\left(x_{1}, \bar{x}\right)
$$

where $\bar{x}_{i}:=x_{i}-x_{1}$ for $i=2, \ldots, N$ and $\bar{x}:=\operatorname{Col}\left(\bar{x}_{2}, \ldots, \bar{x}_{N}\right)$. As a result of the transformation $\left(A_{N}, B_{N}, C_{N}, D_{N}, E_{N}\right) \mapsto\left(A_{o}, B_{o}, C_{o}, D_{o}, E_{o}\right)$ where

$$
\begin{aligned}
& A_{o}:=T\left(I_{N} \otimes A\right) T^{-1}=\left(I_{N} \otimes A\right) \\
& B_{o}:=T\left(I_{N} \otimes B\right)=\left(T_{s}^{-1} \otimes B\right)=\left(I_{N} \otimes B\right)\left(T_{s}^{-1} \otimes I_{m}\right)(14) \\
& D_{o}:=T\left(I_{N} \otimes D\right)=\left(T_{s}^{-1} \otimes D\right)=\left(I_{N} \otimes D\right)\left(T_{s}^{-1} \otimes I_{q}\right) \\
& E_{o}:=T\left(I_{N} \otimes E\right)=\left(T_{s}^{-1} \otimes E\right)=\left(I_{N} \otimes E\right)\left(T_{s}^{-1} \otimes I_{r}\right)
\end{aligned}
$$

and $C_{o}=C_{N} T^{-1}=(L \otimes C)\left(T_{s} \otimes I_{n}\right)=\left(L T_{s} \otimes C\right)$ In order to decouple the relative sensing signal, scale $z$ by $\left(T_{s}{ }^{\mathrm{T}} \otimes I_{p}\right)$ to create

$$
z_{o}:=\left(T_{s}^{\mathrm{T}} \otimes I_{p}\right) z=\left(T_{s}^{\mathrm{T}} \otimes I_{p}\right)\left(L T_{s} \otimes C\right) x_{o}=\left(T_{s}^{\mathrm{T}} L T_{s} \otimes C\right) x_{o}
$$

From the definition of $T_{s}$ and the row-sum-equal-to-zero property of $L$ it is easy to check

$$
T_{s}{ }^{\mathrm{T}} L T_{s}=\left[\begin{array}{cc}
0 & 0 \\
0 & L_{r}
\end{array}\right]
$$

where $L_{r} \in \mathbb{R}^{(N-1) \times(N-1)}$ and in fact the matrix $L_{r}$ in (18) is a sub-matrix of the original Laplacian matrix with entries obtained by setting the entire first column and row to zero. In
(18), $L_{r}$ is a symmetric positive definite matrix. The scaled relative output measurements in the new coordinate system are

$$
z_{o}=\left[\begin{array}{c}
0 \\
\left(L_{r} \otimes C\right)
\end{array}\right] \bar{x}
$$

From the definition in (14)

$$
B_{o} u=\left(I_{N} \otimes B\right)\left(T_{s}^{-1} \otimes I_{m}\right) u=\left(I_{N} \otimes B\right) \underbrace{\mathcal{C} o l\left(u_{1}, \bar{u}\right)}_{u_{o}}
$$

and

$$
D_{o} f(t)=\left(I_{N} \otimes D\right)\left(T_{s}^{-1} \otimes I_{q}\right) f(t)=\left(I_{N} \otimes D\right) \underbrace{\mathcal{C o o l}\left(f_{1}, \bar{f}\right)}_{f_{o}}
$$

where $\bar{u}=\operatorname{Col}\left(\bar{u}_{2}, \ldots, \bar{u}_{N}\right)$ with $\bar{u}_{i}=u_{i}-u_{1}$ and $\bar{f}=$ $\operatorname{Col}\left(\bar{f}_{2}, \ldots, \bar{f}_{N}\right)$ with $\bar{f}_{i}=f_{i}-f_{1}$ for $i=2, \ldots, N$. Define $\bar{z}=\left(L_{r} \otimes C\right) \bar{x}$ then by considering the last $(N-1) n$ rows of the partitioned structure of $x_{o}, u_{o}$ and $z_{o}$, an observable sub-system can be written as

$$
\begin{aligned}
\dot{\bar{x}}(t)= & \underbrace{\left(I_{N-1} \otimes A\right)}_{\bar{A}} \bar{x}(t)+\underbrace{\left(I_{N-1} \otimes B\right)}_{\bar{B}} \bar{u}(t)+\underbrace{\left(I_{N-1} \otimes D\right)}_{\bar{D}} \bar{f}(t) \\
& +\underbrace{\left(I_{N-1} \otimes E\right)}_{\bar{E}} \bar{\xi}(t) \\
\bar{z}(t)= & \underbrace{\left(L_{r} \otimes C\right)}_{\bar{C}} \bar{x}(t)
\end{aligned}
$$

where $\bar{\xi}:=\left(T_{s}^{-1} \otimes I_{r}\right) \xi$ and $\bar{\xi}=\operatorname{Col}\left(\bar{\xi}_{2}, \ldots, \bar{\xi}_{N}\right)$.

Lemma 2: The pair $\left(L_{r} \otimes C, I_{N-1} \otimes A\right)$ is observable.

Proof: Using arguments similar to those in the proof of Lemma 1, it can be verified the pair $\left(\left(L_{r} \otimes C\right),\left(I_{N-1} \otimes A\right)\right)$ is observable because all the eigenvalues of $L_{r}$ matrix are positive.

Spectral decomposition of symmetric matrix $L_{r}$ yields

$$
L_{r}=\bar{V} \bar{\Lambda} \bar{V}^{\mathrm{T}}
$$

where $\bar{\Lambda}=\operatorname{Diag}\left(\bar{\lambda}_{2}, \ldots, \bar{\lambda}_{N}\right)$ are the eigenvalues s.t. $\bar{\lambda}_{i}>$ $0, \forall i=2 \ldots N$, and $\bar{V} \in \mathbb{R}^{(N-1) \times(N-1)}$ is an orthogonal matrix of the associated eigenvectors. Define a change of coordinates $\bar{x} \rightarrow \bar{T} \bar{x}=\tilde{x}$ where $\bar{T}:=\left(\bar{V}^{\mathrm{T}} \otimes I_{n}\right)$. In the $\bar{x}$ coordinates the system matrices $(\bar{A}, \bar{B}, \bar{D}, \bar{E}) \mapsto(\tilde{A}, \tilde{B}, \tilde{D}, \tilde{E})$ where

$$
\begin{aligned}
& \tilde{A}:=\bar{T}\left(I_{N-1} \otimes A\right) \bar{T}^{-1}=\left(I_{N-1} \otimes A\right) \\
& \tilde{B}:=\bar{T}\left(I_{N-1} \otimes B\right)=\left(\bar{V}^{\mathrm{T}} \otimes B\right)=\left(I_{N-1} \otimes B\right)\left(\bar{V}^{\mathrm{T}} \otimes I_{m}\right)(24) \\
& \tilde{D}:=\bar{T}\left(I_{N-1} \otimes D\right)=\left(\bar{V}^{\mathrm{T}} \otimes D\right)=\left(I_{N-1} \otimes D\right)\left(\bar{V}^{\mathrm{T}} \otimes I_{q}\right)(25) \\
& \tilde{E}:=\bar{T}\left(I_{N-1} \otimes E\right)=\left(\bar{V}^{\mathrm{T}} \otimes E\right)=\left(I_{N-1} \otimes E\right)\left(\bar{V}^{\mathrm{T}} \otimes I_{r}\right)(26)
\end{aligned}
$$

Define $\tilde{u}:=\left(\bar{V}^{\mathrm{T}} \otimes I_{m}\right) \bar{u}$ and $\tilde{f}(t):=\left(\bar{V}^{\mathrm{T}} \otimes I_{q}\right) \bar{f}(t)$ and scale $\bar{z}$ using $\left(\bar{\Lambda}^{-1} \bar{V}^{\mathrm{T}} \otimes I_{p}\right)$ to create

$$
\tilde{z}:=\left(\bar{\Lambda}^{-1} \bar{V}^{\mathrm{T}} \otimes I_{p}\right) \bar{z}
$$

then it follows $\tilde{z}:=\left(\bar{\Lambda}^{-1} \bar{V}^{\mathrm{T}} \otimes I_{p}\right)\left(L_{r} \otimes C\right)\left(\bar{V} \otimes I_{n}\right) \tilde{x}=$ $\underbrace{\left(I_{N-1} \otimes C\right)}_{\tilde{C}} \tilde{x}$. Thus, in the new coordinate system (20) - (21) is equivalent to the decoupled systems

$$
\begin{aligned}
& \dot{\tilde{x}}_{i}(t)=A \tilde{x}_{i}(t)+B \tilde{u}_{i}(t)+D \tilde{f}_{i}(t)+E \tilde{\xi}_{i}(t) \\
& \tilde{z}_{i}(t)=C \tilde{x}_{i}(t)
\end{aligned}
$$

for $i=2, \ldots, N$ where $\mathcal{C} \operatorname{Col}\left(\tilde{\xi}_{2}, \ldots, \tilde{\xi}_{N}\right):=\tilde{\xi}$ and $\tilde{f}_{i}(t)=$ $\sum_{j=1}^{N-1} \bar{v}_{j i} \bar{f}_{j}(t)$ where $\bar{v}_{i j}$ is the $i, j$ th element of $\bar{V}$. 


\section{SLIDING MODE OBSERVER DESIGN}

The $N-1$ decoupled dynamical systems in (28)-(29) will be used as the basis for the design of sliding mode observers to reconstruct (initially) the signals $\tilde{f}_{i}$ using the approach described in [27]. In sliding mode observers the state estimation error is constrained to evolve along a surface associated with forcing the output estimation error to be identically zero. The resulting motion - the so-called sliding mode - is of lower dynamical order, and has the property of invariance to a certain class of uncertainty [24]. It is assumed that the states of the system $\tilde{x}_{i}$ and the signals $\tilde{f}_{i}(t)$, which can be viewed as 'virtual faults', are unknown. Only the signals $\tilde{u}_{i}$ and $\tilde{z}_{i}$ from (28)-(29) are available to a centralized scheme and can be obtained by transformations of the control signals $u$ and relative measurements $z$.

For each subsystem of the form given in (28)-(29) an observer of the form

$$
\dot{\tilde{w}}_{i}=A \tilde{w}_{i}+B \tilde{u}_{i}-G_{l} e_{z_{i}}+G_{n} \nu_{i}
$$

can be created where the local output estimation error $e_{z_{i}}:=$ $C \tilde{w}_{i}-\tilde{z}_{i}$ and $\tilde{z}_{i}$ is the relative measurement output from (29). The nonlinear injection term in (30) is given by

$$
\nu_{i}=-\rho_{i}\|C D\| \frac{e_{z_{i}}}{\left\|e_{z_{i}}\right\|}, \quad \text { if } e_{z_{i}} \neq 0
$$

and depends only on $e_{z_{i}}$. The modulation scalar $\rho_{i} \in \mathbb{R}_{+}$must be chosen to satisfy $\rho_{i}>\beta_{0}+\eta$ for some positive scalar $\eta$. The gains $G_{l}$ and $G_{n}$ must be computed to ensure a stable sliding motion on the surface $\mathcal{S}=\left\{\left(e_{2}, \ldots, e_{N}\right) \mid C e_{i}=\right.$ 0 for all $i=2 \ldots N\}$. If the uncertainty $\xi_{i}=0$, necessary and sufficient conditions for the existence of the gains $G_{l}$ and $G_{n}$ such that the state estimates $\tilde{w}_{i}(t) \rightarrow \tilde{x}_{i}(t)$ as $t \rightarrow \infty$ despite the presence of the faults $\tilde{f}_{i}$, is that Assumptions 2-4 hold [28]. An estimate for $\tilde{f}_{i}$ written as $\tilde{f}_{i}^{\text {est }}$, are then obtained as

$$
\tilde{f}_{i}^{e s t}=W \nu_{e q, i}, \quad W \in \mathbb{R}^{q \times m}
$$

for an appropriate choice of $W$ where $\nu_{e q, i}$ is the socalled equivalent injection signal associated with the signal in (31), and represents the average value of the high frequency switched component $\nu_{i}$ required to maintain a sliding motion. For a more detailed explanation see [27]. Since $\nu_{e q, i}$ 'compensates' for the unknown inputs to maintain sliding, it is logical that proper interrogation of the signal $\nu_{e q, i}$ yields information about $\tilde{f}_{i}$. Here, as in (32), the estimate $\tilde{f}_{i}^{\text {est }}$ is chosen as a linear combination of the components of $\nu_{e q, i}$ (which can be obtained by low-pass filtering of $\nu_{i}$ or by approximating (31) by a sigmoidal signal [27]). Formally the gains $G_{l}, G_{n}$ and $W$ can be synthesised to minimize the $\mathcal{L}_{2}$ gain between $\tilde{\xi}_{i}$ and the estimation error $\tilde{f}_{i}^{e s t}-\tilde{f}_{i}$. This problem can be efficiently solved using LMIs [28].

Figure 1 shows the block schematic of the plant and observer structure at a node level. Note that the available 'real' control signals undergo the transformation $u \in \mathbb{R}^{N m} \mapsto$ $u_{o} \in \mathbb{R}^{N m}$ followed by a projection operation to provide $\bar{u} \in \mathbb{R}^{(N-1) m}$. Another transformation followed by demultiplexing provides the $\tilde{u}_{i} \in \mathbb{R}^{m}$ for $i=2 \ldots N$ for use as the available signal in (30).

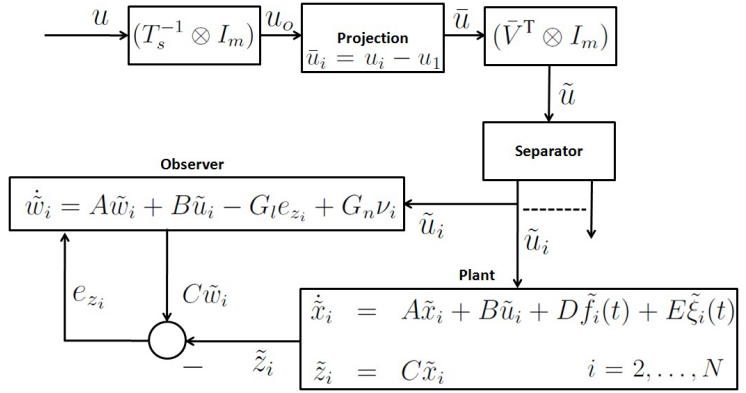

Fig. 1. Observer architecture

The estimate of the virtual fault at the network level is

$$
\tilde{f}^{e s t}(t):=\left(I_{N-1} \otimes W\right) \nu_{e q}
$$

where $\tilde{f}^{\text {est }}:=\operatorname{Col}\left(\tilde{f}_{1}^{e s t}, \ldots, \tilde{f}_{N-1}^{e s t}\right)$ and $\nu_{e q}:=$ $\operatorname{Col}\left(\nu_{e q_{2}}, \ldots, \nu_{e q_{N}}\right)$. To obtain $\bar{f}^{e s t}$, i.e the estimates of the relative faults $\bar{f}$, the inverse transformation

$$
\bar{f}^{e s t}(t):=\left(\bar{V} \otimes I_{q}\right) \tilde{f}^{e s t}(t)
$$

is employed and hence

$$
\bar{f}_{i+1}^{e s t}(t)=\sum_{j=1}^{N-1} \bar{v}_{i j} W \nu_{e q_{j}}
$$

for $i=1, \ldots, N-1$ where $\bar{v}_{i j}$ is the $i, j$ element of $\bar{v}$. The expression in (35) reconstructs the relative faults. (Recall that $\bar{f}_{i}(t)=f_{i}(t)-f_{1}(t)$.) Figure 2 explains the fault estimation. The original faults can then be reconstructed by appropriate logic as outlined below:

i) Case 1: When there is no fault (i.e $f_{1}(t) \equiv 0$ ) in node 1: Since by definition $\bar{f}_{i}(t)=f_{i}(t)-f_{1}(t)$, when $f_{1}(t)=0$ it follows $f_{i}(t)=\bar{f}_{i}(t)$ for $i \geq 2$ and is estimated by $\bar{f}_{i}^{e s t}(t)$.

ii) Case 2: When there is a fault in $f_{1}(t)$ and no faults exist in any other nodes $f_{i}(t)=0$ for $i=2 \ldots N$. Since $\bar{f}_{i}(t)=f_{i}(t)-f_{1}(t)$, it follows $\bar{f}_{i}(t)=-f_{1}(t)$ for $i=2, \ldots, N$ and so all reconstructions $\bar{f}_{i}^{e s t}(t)$ for $i=2, \ldots, N$ provide fault estimations for $-f_{1}(t)$.

iii) Case 3: When there are multiple faults, the situation cannot necessarily be described generically, and fault isolation though estimation will be more difficult. However, for example, consider the case when a fault occurs in nodes 1 and $k$ so that $f_{1}(t) \neq 0$ and $f_{k}(t) \neq 0$. As argued above $f_{1}(t)$ will affect $\bar{f}_{i}^{\text {est }}(t)$ for all $i=2, \ldots, N$. Furthermore, the fault $f_{k}(t)$ will appear in $\bar{f}_{k}^{e s t}(t)$ only - specifically $\bar{f}_{k}(t):=f_{k}(t)-f_{1}(t)$ and there will be a 'cumulative effect'. However, $f_{1}(t)$ and $f_{k}(t)$ can still be estimated sequentially using appropriate logic. In multiple faults in nodes other than the $1^{\text {st }}$ one, the logic is easier since there is no cumulative effect and $\bar{f}_{i}^{\text {est }}(t)=f_{i}$ and $\bar{f}_{j}^{e s t}(t)=f_{j}$ for $i, j \in\{2, \ldots, N\}$.

Remark 2: For a network with $N$ nodes, the proposed observer framework has $N-1$ observers, and each observer is designed in a decentralised manner in the coordinates of (28)(29). This provides computational tractability in the case of large scale systems of high order and many nodes. However, 


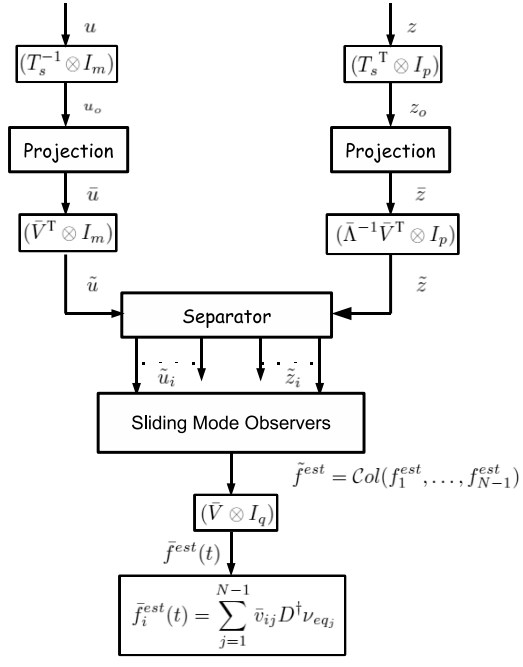

Fig. 2. Fault reconstruction architecture

the actual implementation of the observers for reconstruction of the faults, requires a centralised approach.

Remark 3: The centralised approach described above could be prohibitive in the case of a very large network, and in such a situation semi-decentralised, decentralised and/or distributed approaches would be more beneficial. However the ideas above can also be used to create a semi-decentralised observer architecture similar to [21]. First define $N_{i}=\left|\mathcal{F}_{i}\right|$ and let $\left\{j_{1}, j_{2} \ldots, j_{N_{i}}\right\}=\mathcal{F}_{i}$ i.e., the indices of the nodes for which node $i$ has relative information. Define an augmented state by

$$
\bar{x}_{i}=\operatorname{Col}\left(x_{i}, x_{j_{1}}, x_{j_{2}} \ldots x_{j_{N_{i}}}\right)
$$

Suppose that each node has a control law $u_{i}=K x_{i}$, then

$$
\dot{\bar{x}}_{i}(t)=\bar{A} \bar{x}_{i}(t)+\bar{D} \bar{f}_{i}(t)
$$

where $\bar{f}_{i}=\operatorname{col}\left(f_{i}, f_{j_{1}}, f_{j_{2}} \ldots, f_{j_{N_{i}}}\right)$ and the matrices $\bar{A}=$ $\left(I_{N_{i}+1} \otimes A_{c}\right)$ and $\bar{D}=\left(I_{N_{i}+1} \otimes D\right)$ where $A_{c}=A+B K$. Suppose that instead of summing the relative information $z_{i j}$ as in (3), the information is concatenated as

$$
\bar{z}_{i}=\operatorname{Col}\left(z_{i j_{1}}, z_{i j_{2}} \ldots, z_{i j_{N_{i}}}\right)
$$

The information in $\bar{z}_{i} \in \mathbb{R}^{q N_{i}}$ is available at node $i$ and $\bar{z}_{i}=$ $\bar{C} \bar{x}_{i}$ where

$$
\bar{C}=\left[\begin{array}{cccccc}
C & -C & 0 & 0 & 0 & 0 \\
C & 0 & -C & 0 & 0 & 0 \\
\vdots & \vdots & \ldots & 0 & -C & 0 \\
C & 0 & 0 & 0 & 0 & -C
\end{array}\right]
$$

For the system associated with the triple $(\bar{A}, \bar{D}, \bar{C})$ of order $n\left(N_{i}+1\right)$, it can be shown using a similar approach to that employed in Section II, that the pair $(\bar{A}, \bar{C})$ is unobservable, but by applying the state transformation in Lemma 1 , an observable $n N_{i}$ order system can be identified of the form $\left(\left(I_{N_{i}} \otimes A_{c}\right),\left(I_{N_{i}} \otimes D\right),\left(I_{N_{i}} \otimes C\right)\right)$ associated with relative faults $\tilde{f}_{j}=f_{j}-f_{i}$ for $j \in \mathcal{F}_{i}$. If the triple $(A, D, C)$ satisfies Assumptions 2-4 then $\left(A_{c}, D, C\right)$ also satisfies Assumptions 2-4 and the sliding mode methodology described above is directly applicable to estimate the relative faults $\tilde{f}_{j}$. If $N_{i}>2$ then the actual faults can be reconstructed as described in Section III. Note that the resulting semi-decentralised observer deployed at node $i$ only requires the measurements in (38) which are local and available at that node.

\section{Simulations}

A network of twenty five interconnected flexible link robot dynamical systems is considered, each modelled as [29], [30]:

$$
\begin{aligned}
{\left[\begin{array}{c}
\dot{\theta}_{m}^{i} \\
\dot{\omega}_{m}^{i} \\
\dot{\theta}_{l}^{i} \\
\dot{\omega}_{l}^{i}
\end{array}\right]=} & {\left[\begin{array}{cccc}
0 & 1 & 0 & 0 \\
-\frac{K_{l}}{J_{m}} & -\frac{B}{J_{m}} & \frac{K_{l}}{J_{m}} & 0 \\
0 & 0 & 0 & 1 \\
\frac{K_{l}}{J_{l}} & 0 & -\frac{K_{l}}{J_{l}}-\frac{m g h}{J_{l}} & 0
\end{array}\right]\left[\begin{array}{c}
\theta_{m}^{i} \\
\omega_{m}^{i} \\
\theta_{l}^{i} \\
\omega_{l}^{i}
\end{array}\right]+} \\
& {\left[\begin{array}{c}
0 \\
\frac{K_{\tau}}{J_{m}} \\
0 \\
0
\end{array}\right] u^{i}+\left[\begin{array}{c}
0 \\
\frac{K_{\tau}}{J_{m}} \\
0 \\
0
\end{array}\right] f^{i}+\left[\begin{array}{c}
0 \\
0 \\
0 \\
\frac{m g h}{J_{l}}
\end{array}\right] \xi^{i} } \\
z_{i}= & \sum_{j \in \mathcal{J}_{i}} C\left(x_{i}-x_{j}\right)
\end{aligned}
$$

for $i=1 \ldots N$ where $C=\left[\begin{array}{ll}I_{3} & 0_{3 \times 1}\end{array}\right]$ and the states represent the angular position and velocity of the motor shaft $\left(\theta_{m}^{i}\right.$ and $\left.\omega_{m}^{i}\right)$ and the angular position and velocity of the link $\left(\theta_{l}^{i}\right.$ and $\omega_{l}^{i}$ ). In this example $D=B$ and the scenario which is considered is concerned with an 'actuator fault' occurring in the motor driving the flexible links. The bounded disturbance term $\xi^{i}$ is associated with the gravity term involving approximations of $\sin \left(\theta_{l}^{i}\right)$. Details of the physical parameters in (40) are given in [29], [30]. A randomly generated bidirectional graph Laplacian is used.

Following the transformations discussed in Section II, and depicted in Figure 2, 24 decoupled systems in the form of (28) - (29) are obtained. Then 24 decoupled sliding mode observers, as described in (30), are synthesised following the design procedure described in [28]. The associated observer gains $G_{l_{i}}, G_{n_{i}} \in \mathbb{R}^{4 \times 3}$ and $W \in \mathbb{R}^{1 \times 3}$ are synthesised using LMI optimization to minimize the effect of the $\xi^{i}$,s on the reconstructions [28]. In this example, the gains are

$$
G_{l}=\left[\begin{array}{rrr}
0.1130 & -0.0717 & 0.0472 \\
-0.0581 & 3.2318 & 0.4159 \\
0.0571 & 0.6213 & 0.1416 \\
-0.5567 & 2.1673 & 0.0922
\end{array}\right],
$$

and

$$
G_{n}=\left[\begin{array}{rrr}
1.1296 & -0.5806 & 0.5710 \\
-0.5806 & 26.1774 & 5.0328 \\
0.5710 & 5.0328 & 1.7133 \\
-5.5674 & 17.5551 & 1.1156
\end{array}\right]
$$

and $W=\left[\begin{array}{lll}-0.0269 & 1.2107 & 0.2328\end{array}\right]$. The $\mathcal{L}_{2}$ gain between the uncertainty and the fault estimation error is 0.0463 . Here the nonlinear injection signal in (31) is approximated as $\nu_{e q_{i}}=-\rho_{i} \frac{e_{z_{i}}}{\left\|e_{z_{i}}\right\|+\delta}$ where $\rho=50.0$ and $\delta=0.001$. In the simulations, a variable step integration scheme with the 'Ode $15 \mathrm{~s}$ ' solver is used, with the maximum step-size fixed at 1e-3. Due to space restrictions, a single representative case (multiple faults in the network including node 1) is considered here.

Faults are introduced in the network in the 1st node (a sum of sinusoid occurring at $2.5 \mathrm{sec}$ ), and later in two other nodes (in one node a rate limited staircase fault signal is introduced and then a rate limited sawtooth type signal is introduced in the 

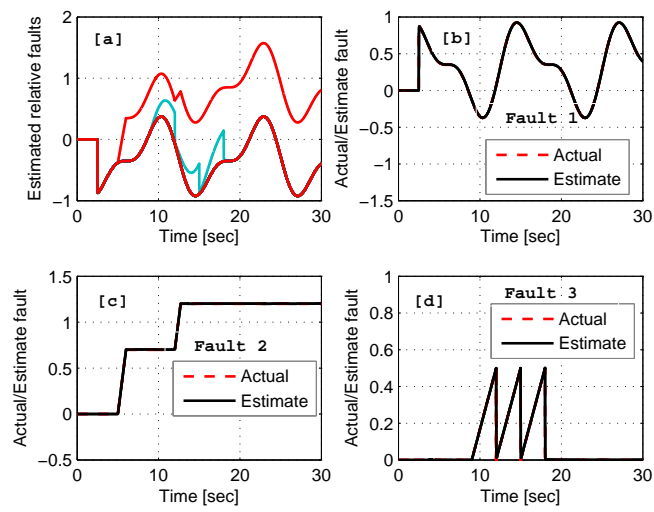

Fig. 3. Fault reconstruction with faults introduced in randomly chosen nodes

other). The strategy is to look at the multiple (nearly) identical reconstruction signals which will correspond to a fault in the first subsystem. Thus, $f_{1}(t)$ is estimated initially. Subsequently all the other faults can be reconstructed by correcting the reconstruction signals so that the estimate of a fault in node $k>1$ is given by $\bar{f}_{k}^{e s t}+f_{1}^{e s t}$ where $f_{1}^{e s t}$ is the estimate of the fault in node 1 . Figure 3 shows a scenario involving the reconstruction of three faults. Figure 3(a) shows all the reconstructed relative fault signals obtained from (35) (24 in this case). Clearly multiple nearly identical signals are present. This is due to the presence of the fault in the first node which is embedded in all the reconstruction signals. In the first subplot of Figure 3, the cumulative effect of the two other faults present in the reconstruction signals can be seen. First $f_{1}(t)$ is estimated (this is shown in the second subplot Figure 3(b)). Subsequently, the effect of the fault in node 1 is 'removed' from all the other reconstruction signals (shown in the last subplot). Note, by doing this, the other two faults are estimated quite accurately as shown in Figure 3(c) and 3(d).

\section{Conclusions}

A robust fault estimation method, based on sliding mode observers, has been proposed for a collection of agents undertaking a shared task and exchanging only relative information over a communication network. The resulting 'system of systems', with respect to relative sensing information, is not observable; however by appropriate transformations and scalings of the inputs and outputs of the actual system, a meaningful observable subsystem can be created. For this new subsystem, after modal decomposition based on the associated Laplacian, an available robust fault estimation scheme can be used to synthesize decentralized observers for fault estimation. These collectively form a centralized fault estimation scheme for the original system. The fault estimation framework proposed in this paper has been applied to a network of flexible link robots to demonstrate its efficacy.

\section{REFERENCES}

[1] J. A. Fax and R. M. Murray, "Information flow and cooperative control of vehicle formations," IEEE Trans. Autom. Control, vol. 49, pp. 14651476, 2004.
[2] P. Massioni and M. Verhaegen, "Distributed control for identical dynamically coupled systems: A decomposition approach," IEEE Trans. Autom. Control, vol. 54, pp. 124-135, 2009.

[3] W. Ren and R. W. Beard, Distributed consensus in multi-vehicle cooperative control. Springer, Lecture notes in Communication and Control Engineering Series, London, 2007.

[4] C. W. Wu, Synchronisation of complex networks of nonlinear dynamical systems. World scientific publishing, Singapore, 2007.

[5] Z. Lin, B. Francis, and M. Maggiore, "State agreement for continuous time coupled nonlinear systems," SIAM J. Control Optim., vol. 46, pp. 288-307, 2007.

[6] A. Azemi and E. Yaz, "Sliding mode adaptive observer for chaotic synchronization,” J. Dyn. Syst-T. ASME, vol. 122, pp. 758-765, 2000.

[7] J. J. Yan, M. Hung, T. Y. Chiang, and Y. Yang, "Robust synchronization of chaotic systems via adaptive sliding mode control," Physica A, vol. 356, pp. 220-225, 2006

[8] Q. Wu and M. Saif, "Robust fault detection and diagnosis for a multiple satellite formation flying system using second order sliding mode and wavelet networks," In Proc. of ACC, New York, 2007.

[9] S. P. M. Noijen, P. F. Lambrechts, and H. Nijmeijer, "An observercontroller combination for a unicycle mobile robot," Int. J. Control, vol. 78, pp. 81-87, 2005.

[10] G. L. Mariottini, G. Pappas, D. Prattichizzo, and K. Daniilidis, "Visionbased localization of leader-follower formations," In Proc. of CDC-ECC, Seville, 2005.

[11] X. G. Yan and C. Edwards, "Robust decentralised actuator fault detection and estimation for large-scale systems using the sliding mode observer," Int. J. Control, vol. 81, pp. 591-606, 2008.

[12] P. Barooah and J. P. Hespanha, "Estimation on graphs from relative measurements: Distributed algorithms and fundamental limits," IEEE Control Syst. Mag., vol. 27, pp. 57-74, 2007.

[13] I. Shames, A. Teixeira, H. Sandberg, and K. H. Johansson, "Distributed fault detection for interconnected second-order systems," Automatica, vol. 47, pp. 2757-2764, 2011.

[14] M. J. Daigle, X. D. Koutsoukos, and G. Biswas, "Distributed diagnosis in formations of mobile robots," IEEE Trans. Robot., vol. 23, pp. 353369, 2007.

[15] W. H. Chung and J. L. Speyer, "A decectralized fault detection filter," in In Proc. of the ACC, Philadelphia, pp. 2017-2021, 1998.

[16] S. Shankar, S. Darbha, and A. Datta, "Design of a decentralized detection filter for a large collection of interacting lti systems," Mathematical Problems in Engineering, vol. 8, pp. 233-248, 2002.

[17] M. F. Hassan, M. A. Sultan, and M. S. Attia, "Fault detection in largescale stochastic dynamic systems," IEE Proc. Part D: Control Theory Appl., vol. 139, pp. 119- 124, 1992.

[18] G. K. A. Rao and N. Viswanadham, "Decentralized fault detection and diagnosis in large linear stochastic systems," J. I. El. Telecom. Eng. vol. 39, pp. 143-148, 1993.

[19] R. J. Patton, C. Kambhampati, A. Casavola, P. Zhang, S. Ding, and A. D. Sauter, "Generic strategy for fault-tolerance in control systems distributed over a network," Eur. J. Control, vol. 13, pp. 280-296, 2007.

[20] C. Kambhampati, C. Perkgoz, R. J. Patton, and W. Ahamed, "An interconnection predictive approach to fault-tolerant control in network control systems," J. Sys and Contr. Eng., vol. 221, pp. 885-894, 2007.

[21] N. Meskin and K. Khorasani, "Actuator fault detection and isolation for a network of unmanned vehicles," IEEE Trans. Autom. Control, pp. 835 $-840,2009$.

[22] M. A. Massoumnia, "A geometric approach to synthesis of failure detection filters," IEEE Trans. Autom. Control, vol. 31, pp. 839-846, 1986.

[23] J. Chen and R. J. Patton, Robust Model-Based Fault Diagnosis for Dynamic Systems. Kluwer Academic Publishers, USA, 1999.

[24] C. Edwards, S. K. Spurgeon, and R. J. Patton, "Sliding mode observers for fault detection," Automatica, vol. 36, pp. 541-553, 2000.

[25] M. Mesbhahi and M. Egerstedt, Graph theoretic methods in multiagent networks. Princeton University Press, Princeton, New Jersey, 2010.

[26] J. M. Maciejowski, Multivariable feedback design. Addison-Wesley, Wokingham, Berkshire, UK, 1989.

[27] C. Edwards and S. K. Spurgeon, Sliding Mode Control: Theory and Applications. Taylor \& Francis, London, 1998.

[28] C. P. Tan and C. Edwards, "Sliding mode observers for robust detection and reconstruction of actuator and sensor faults," Int. J. Robust Nonlin., vol. 13, pp. 443-463, 2003.

[29] S. Raghavan and J. K. Hedrick, "Observer design for a class of nonlinear systems," Int. J. Control, vol. 59, pp. 515-528, 1994.

[30] X. Fan and M. Arcack, "Observer design for systems with multivariable monotone nonlinearities," Syst. Control Lett., vol. 50, pp. 319-330, 2003. 